\title{
Uni-portal and Bi-portal Techniques in Endoscopic Lumbar Spine Surgery: Their Reciprocal Relations
}

\author{
Chun Kun Park \\ Department of Neurosurgery, Good Doctor TeunTeun Hospital, Anyang, Republic of Korea
}

Endoscopic spine surgery (ESS) belongs to minimally invasive spine surgery (MISS). At the early stage in ESS history, most ESS surgeons thought the best indication was a herniated lumbar disc (HLD). Surgeons have done the herniated disc's Endoscopic removal via a transforaminal approach and L5/S1 HLD via an interlaminar approach. Meanwhile, ESS surgeons had not considered the other degenerative lumbar diseases in those days, especially spinal stenosis, as a contraindication. Before a long time, some articles regarding ESS's affirmative results came out and presented efficiency of the endoscopy and good postoperative long-term outcome in well-controlled clinical studies with higher credibility ${ }^{1-3)}$. They also tried to extend ESS indications like spinal stenosis ${ }^{2}$. MISS benefits include minimal collateral damage, minimal scarring, less blood loss, shorter hospital stay, minimal perioperative morbidity ${ }^{4}$, early rehabilitation, higher versatility, nearer visibility, and more enhanced maneuverability ${ }^{5}$ than the other MISS techniques. Since spinal stenosis has become one of the pathologies frequently managed with full endoscopic spine surgery (FESS), many spine institutes ${ }^{5,6)}$ required new designed endoscopic tools and surgery sets. Consequently, its surgical instruments had evolved rapidly.

The ESS surgeons essentially should employ one of the two approaching techniques: UPT or BPT has some differences and similarities. The design of a working-channel endoscope for FESS using a uni-portal method (UPT) changed from a standard long length $(170 \mathrm{~mm})$ and small diameter $(7.3 \mathrm{~mm})$ of the working channel into a new shorter length $(112 \mathrm{~mm})$ and larger diameter $(8.4$ to $9.0 \mathrm{~mm}$ ) one (Techord, Daejon, Korea) within a short time in the comparison between an endoscope for transforaminal route and a new one. One of the critical reasons for the change should be the anatomical (or racial?) differences between Asians and Western. As we know well, most ESS surgical sets are Germanmade. It should be appropriate that German-made endoscopy is inefficient to was Asian surgeons. As a result, these decreasing changes in size brought about efficiency in a surgeon, predominantly Asian, handling hard an endoscope and keeping the endoscopy vertically on the patient's back during the operation. Meantime, endoscopic-assisted spine surgery (E-ASS) using a bi-portal technique (BPT) did not need a new design for an endoscope or surgical apparatuses because of the size of a skin incision of the $2^{\text {nd }}$ portal, usually for the conventional surgical tools. It can develop concerning their sizes and the relatively appropriate length and diameter for the slender non-working channel endoscope.

Only a few spine surgeons had tried to use uni- and bi-portal techniques in lumbar spine pathologies. In the middle of the 2010s, several articles concerning both procedures in spinal stenosis came out. And not a few national and international technical endoscopic workshops became popular in various countries, which motivated spinal surgeons to start ESS using uni- or bi-portal techniques in spinal stenosis patients. Some ESS pioneers had introduced these two approaching techniques almost the same time, and the surgeons, who preferred the one technique, competed with one another. BPT had been remarkably familiar to the orthopedic surgeon because the orthopedics had used BPT or multi-portal techniques in the endoscopic treatment for various joint diseases. As a result, the orthopedic surgeon might have given an idea to use an arthroscope for BPT in degenerative lumbar disorders. UPT and BPT can be useful in dorsal as well as transforaminal approaches. UPT can be applied in FESS, while BPT can be applicable in E-ASS.

There are two techniques to approach the primary lesion at the early or final stage of the operation. A surgeon who tries to remove a pathology including HLD and spinal stenosis, particularly the pathology accessible via intervertebral foramen, should decide one of two techniques, inside-out (IOT) or outside-in (OIT). Most spine surgeons prefer OIT to the other ${ }^{8)}$. The IOT is Yeung's favorite way of approach to a disc lesion, including $H_{L D}{ }^{9)}$. Yeung and some of his followers, who always employ the UPT, still prefer $\left(O T^{10)}\right.$, particularly the HLD removal via the intervertebral foramen ${ }^{11)}$. Practically speaking, the portal's number has nothing to do with patients' outcomes. This issue is validated as long as the primary lesion is accessible via the intervertebral foramen. We need to understand the principal running place of an ordinary endoscope should be an intervertebral foramen.

In 2020, Hofstetter and 27 endoscopic spine surgeons developed ESS consensus nomenclature under the AOSpine MISS taskforce $^{10)}$. They reported a list of terminology related to ESS. They suggested each endoscopic procedure's name by a systematic

Corresponding Author: Chun Kun Park, MD, PhD

Department of Neurosurgery, Good Doctor TeunTeun Hospital, 775 Kyeongsu-daero, Anyang-si Kyeonggi-do, Republic of Korea

TEL: +82-32-8086-8357, E-mail: ckpmd@catholic.ac.kr 
nomenclature system in regular sequence and consistent combination of words. This consensus nomenclature system may not be an undisputed guideline all ESS surgeons should follow. Some ESS surgeons had gotten a name different from the rule of AOSpine nomenclature ${ }^{12)}$. A surgeon should differentiate the UPT from a hybrid operation (mini-open/tubular/bi-portal technique $)^{10}$. These authors also presented that BPT is irrelevant to FESS but relevant to E-ASS. A surgeon can apply the UPT in all the FESS.

It may be a wonder if the approaching technique's difference substantially influences surgical result and outcome. According to the published articles, there were positive results of the clinical variables, which presented postoperatively well decrease of VAS, well increase of the surgical results, good outcome (increased ODI), an increase of satisfaction, disclosed no significant difference between two techniques. In a comparison study to microdecompression in the patients with spinal stenosis, ESS showed better results regardless of uni- or bi-portal technique ${ }^{10,13)}$. The extent of collateral damage, including muscle injury, demonstrated the largest microdecompression ${ }^{14)}$ and least in FESS ${ }^{6,14)}$. E-ASS showed an increase of muscle damage instead compared to FESS but statistically not significant. This result seems to be no wonder because a working space would be more significant in BPT, using two apparatuses together, which needs a larger room in the muscle-lamina interface. As a result, more blood loss could be more prominent in BPT compared to UPT. Both techniques have another advantage: facet preservation, so-called facet undercutting, or medial facetectomy, leading to segmental stability ${ }^{6)}$.

Conclusively, UPT and BPT both techniques demonstrated similar clinical results and outcomes. However, UPT is employed only in FESS, and BPT is only in E-ASS. Each technique has its purpose and specific condition to apply appropriately. Inside-out and outside-in should be involved only in the transforaminal approach $^{10)}$. ESS surgeon needs to understand what the unfamiliar terminology stands for.

\section{CONFLICT OF INTEREST}

No potential conflict of interest relevant to this article.

\section{REFERENCES}

1. Komp M, Hahn P, Oezdemir S, et al.: Bilateral spinal decompression of lumbar central stenosis with the full-endoscopic interlaminar versus microsurgical laminotomy technique: A prospective, randomized, controlled study. Pain Physician 18:61-70, 2015
2. Ruetten S, Komp M, Merk H, Godolias G: Surgical treatment for lumbar lateral recess stenosis with the full-endoscopic interlaminar approach versus conventional microsurgical technique: A prospective, randomized, controlled study. J Neurosurg Spine 10:476-485, 2009

3. Gibson JNA, Subramanian AS, Scott CEH: A randomised controlled trial of transforaminal endoscopic discectomy vs microdiscectomy. Eur Spine J 26:847-856, 2017

4. Pan L, Zhang P, Yin Q: Comparison of tissue damages caused by endoscopic lumbar discectomy and traditional lumbar discectomy: A randomised controlled trial. Int J Surg 12:534-535, 2014

5. Akbary K, Kim J-S, Park CW, Jun SG, Hwang JH: Biportal endoscopic decompression of exiting and traversing nerve roots through a single interlaminar window using a contralateral approach: Technical feasibilities and morphometric changes of the lumbar canal and foramen. World Neurosurg 117:153-161, 2018

6. Heo DH, MD, Lee DC, MD, Park CK: Comparative analysis of three types of minimally invasive decompressive surgery for lumbar central stenosis: Biportal endoscopy, uniportal endoscopy, and microsurgery. Neurosurg Focus 46(5):E9, 2019

7. Pranata R, Lim MA, Vania R, Julius July J: Biportal Endoscopic Spinal Surgery versus Microscopic Decompression for Lumbar Spinal Stenosis: A Systematic Review and Meta-Analysis. World Neurosurg 138:e450-e458, 2020

8. Schubert M, Hoogland T: Endoscopic transforaminal nucleotomy with foraminoplasty for lumbar disk herniation. Oper Orthop Traumatol 17:641-661, 2005

9. Yeung AT: Minimally invasive disc surgery with the Yeung Endoscopic Spine System (YESS). Surg Technol Int. 8:267-277, 1999

10. Hofstetter CP, Ahn Y, Choi G, et al.: AOSpine consensus paper on nomenclature for working-channel endoscopic spinal procedures. Global Spine Journal 10(2S):111S-121S, 2020

11. Gibson JN,Cowie JG, Iprenburg M: Transforaminal endoscopic spinal surgery: The future "gold standard" for discectomy? A review. Surgeon 10:290-296, 2012

12. Lim KT, Nam HGW, Kim SB, Kim HS, Park JS, Park CK: Therapeutic feasibility of full endoscopic decompression in one- to three-level lumbar canal stenosis via a single skin port using a new endoscopic system, percutaneous stenoscopic lumbar decompression. Asian Spine J Apr;13(2):272-282, 2019 doi: 10. 31616/asj. 2018.0228. Epub Nov; 27.PMID:30472819, 2018

13. Choi K-C, Shim H-K, Hwang J-S, Shin SH, Lee DC, Jung HH, Park HA, Park CK: Comparison of surgical invasiveness between microdiscectomy and 3 different endoscopic discectomy techniques for lumbar disc herniation. World Neurosurg 116:e750-e758, 2018

14. Garg B, Nagraja UB, Jayaswal A: Microendoscopic versus open discectomy for lumbar disc herniation: A prospective randomized study. J Orthop Surg (Hong Kong) 19:30-34, 2011 